\title{
Review on Transdermal Drug Delivery Systems
}

\section{Pang $\mathrm{Z}$ and Han $\mathrm{C}^{*}$}

School of Pharmacy, Shandong University of Traditional Chinese Medicine, Jinan 250355, People's Republic of China ${ }^{*}$ Corresponding author: Han C, School of Pharmacy, Shandong University of Traditional Chinese Medicine, Jinan 250355, People’s Republic of China, E-mail: pangzunting@126.com

Citation: Pang Z, Han C (2014) Review on Transdermal Drug Delivery Systems. J Pharm Drug Devel 2(4): 402. doi: 10.15744/2348-9782.1.602

Received Date: September 16, 2014 Accepted Date: December 18, 2014 Published Date: December 22, 2014

\begin{abstract}
Recently, transdermal drug delivery system (TDDS) has become a more and more important approach to administering drugs. Based on its advantages, which are not achievable by other modes of administration, many researchers are dedicated to the study of it, and have made great progress. Although the skin offers a painless interface for systemic drug delivery, it also presents limitations which are mainly caused by the stratum corneum. In this work, we state the increasingly impact of TDDS, discuss the limitations of it, and last but not least, we highlight the methods for overcoming these limitations by using permeation enhancers, microneedles, iontophoresis.
\end{abstract}

Keywords: Transdermal drug delivery system; Permeation enhancers; Iontophoresis; Microneedles

\section{Introduction}

During the past decades, intensive studies have focused on the technologies in drug delivery, and with the rapid developments and explorations in technologies, traditional drug delivery means are being replaced by the more effective and advanced ones. The creation of transdermal drug delivery system (TDDS) has been one of the most sophisticated and innovative approaches of drug deliveries. The transdermal drug delivery system has attracted considerale attention because of its many potential advantages, including better patient compliance, avoidance of gastrointestinal disturbances, hepatic first-pass metabolism and sustained delivery of drugs to provide steady plasma profiles, particularly for drugs with short half-lives, reduction in systemic side effects and enhanced therapeutic efficacy [1-6].

Despite these advantages, most of the transdermal candidates have low permeability. The drugs administered across skin should have the three constraining characteristics: appropriate partition coefficient, low molecular mass $(<500 \mathrm{Da})$, and small required dose (upto milligrams) [3]. The limitations of transdermal drug delivery are caused by skin which protects against and is impermeable to foreign molecules. The human skin is consisted of two main layers: the layer of epidermis and the layer of dermis. Stratum corneum is the epidermis's outermost layer that composed of stratified keratinocytes, multiple lipid bilayers of ceramidas, fatty acids, cholesterol and cholesterol esters. Stratum corneum provides an extremely effective physical barrier for the control of drug penetration [2,7-10]. Therefore, attempts to overcome this skin barrier is presently an important area of pharmaceutical and toxicological research. The techniques that weaken the barrier have included permeation enhancers [11-43], microneedles [44-68] and iontophoresis [69-84].

\section{Chemical permeation enhancers}

In the last 50 years, a large number of chemical permeation enhancers (CPEs) which are defined as substances that interact with the major constituents of skin barrier, stratum corneum, to promote penetration of drugs into skin.

The ideal enhancer should have the following conditions:

1. Non-pharmacological activities.

2. Nontoxic, non-allergenic, and non-irritating.

3. Rapid-acting with predictable and reproducible activity.

4. When removed from the skin surface, the penetrability of the skin should recover immediately.

5. Cosmetically acceptable with suitable skin feel [11].

The part of penetration enhancer in topical formulations has been becoming significantly and undoubtedly, and they would permit the delivery of broader classes of drugs through the stratum corneum in the future. 


\section{The classification of chemical permeation enhancers}

From the above mentioned, we can know that the skin provides such a formidable obstacle to the delivery of most drugs, a variety of different chemical permeation enhancers have been tested to enhance transdermal penetration. Immense amounts of research during the past two decades has led to the formulation of some different classes of penetration enhancers, including surfactants (for example, sorbitan trioleate), fatty acids/esters (for example, lauric acid), terpenes (for example, menthone), azone-like compounds (for example, azone), and solvents (for example, ethanol and dimethyl sulphoxide) (Table 1) [1,6,12-15].

\begin{tabular}{|l|l|}
\hline \multicolumn{1}{|c|}{ Types } & \multicolumn{1}{c|}{ Examples } \\
\hline Surfactants & $\begin{array}{l}\text { Sorbitan monopalmitate, Sorbitan trioleate, } \\
\text { Cetyl trimethyl ammonium bromide }\end{array}$ \\
\hline Fatty acids/esters & $\begin{array}{l}\text { Alkanoic acids, oleic acid, lauric acid, capric acid, Cetyl lactate, } \\
\text { butyl acetate, isopropyl myristate }\end{array}$ \\
\hline Terpenes & Nerolidol, Farnesol, Carvone, Menthone \\
\hline Azone-like compounds & $\begin{array}{l}\text { Azone (Laurocapram, 1-dodecylazacycloheptan-2-one), } \\
\text { 1-alkyl- or 1-alkenylaza cycloalkanones }\end{array}$ \\
\hline Solvents & DMSO, ethanol, 1-octanol, 1-hexanol \\
\hline
\end{tabular}

Table 1: Types of chemical penetration enhancers

Fatty acids for the use of skin permeation enhancers: Fatty acids contain a aliphatic hydrocarbon chain and a terminal carboxylic acid group. Because the number, position, and configuration of double bonds of their aliphatic chain, which is either saturated or un-saturated, are visible different, and the aliphatic chain may have branching and other substituents, fatty acids have different characteristics and enhancing effects [16,17].

A study of Florence et al. indicates that with the increasing of the carbon chain length, the lipophilicity of fatty acids also increases. So long-chain fatty acids have a much higher affinity to lipids in the stratum corneum, while short-chain fatty acids have insufficient lipophilicity which goes against the skin penetration [18].The work of Guy et al. shows that the stratum corneum of the human skin is a lipophilic organizational structure and that compounds of high lipophilicity tend to remain in the SC, preventing drugs to transport to the deeper tissues [19]. Evidences from many studies have confirmed that the enhancing effects of fatty acids were most significant for C10 to C12 fatty acids [20,21].

In addition, the enhancing effects of fatty acid was also influenced by the bond saturation. Some studies have demonstrated that the unsaturated long-chain fatty acids have been shown a greater enhancer activity when compared to saturated fatty acids of the same chain length. This is mainly attributed to the higher disrupting nature of the unsaturated long-chain of these fatty acids that would result in a higher magnitude of lipid disruption [22,23].

Last but not least, the enhancement effects of fatty acids on penetration through the stratum corneum are also dependent on the concentrations of fatty acids. Some studies have shown that the skin permeation of Meloxicam through human skin increased when the concentration of oleic acid increased from 0.4 to $1 \%$.

Terpenes for the use of skin permeation enhancers: Terpenes are a series of volatile oil from natural sources or laboratory designed, and consist of repeated isoprene $\left(\mathrm{C}_{5} \mathrm{H}_{8}\right)$ units, in a head-to-tail orientation to form linear chains or rings, which is the basic chemical structure of terpenes [24,25].

Terpenes, as a kind of clinically acceptable penetration enhancers have the following advantages:

1) When compared to other synthetic skin penetration enhancers, terpenes are generally less toxicity with low irritancy. And quite a few terpenes have been classified as generally regarded as safe by the Food and Drug Administration (FDA) [26-30].

2) Reversible effect on the lipids of stratum corneum [31].

During the past two decades, the satisfactory performance of terpenes in enhancing the permeation of drug through the skin has been intensively revealed. Terpenes can increase skin permeation by interacting with SC lipids and keratin, and increasing the solubility of drug into SC lipids [26]. There are some main factors that play an important role in the permeation enhancing activity of terpenes:

1. The chemical structures of terpenes: It has been commonly observed that terpene stereoisomers can affect their enhancing activity. Some researcher have reported that the (-) enantiomer of a terpene is more effective than the corresponding ( \pm ) racemate or the $(+)$ isomer. Futhermore, some terpenes with a minimal degree of saturation are more effective enhancers to deliver the hydrophilic drugs [32,33]. 
2. The lipophilicity of terpenes: High lipophilicity is an important structural feature for terpenes; accordingly, hydrocarbon, nonpolar terpenes, such as limonene, are more effective to promote the transdermic absorption of lipophilic drugs than oxygencontaining polar terpenes and vice versa $[34,35]$.

3. The physical form of terpenes: From some studies, we can know that the liquid terpenes have a greater ability to form hydrogen bonds with intercellular lipids of SC, so liquid terpenes can produce better enhancing effects than the solid ones [36].

Azone-like compounds for the use of skin permeation enhancers: Azone, see Figure 1, is a highly lipophilic substance with a $\log \mathrm{P}$ around 6.2, and it can be compatible with many types of organic solvents, such as alcohols and propylene glycol. Besides, Azone also has low irritancy and very low toxicity [37].

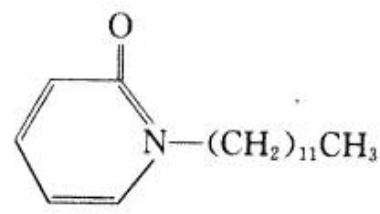

Figure 1: The structure of laurocapram (1-dodecylazacykloheptan-2-one, laurocapram,6)

In the last few years, Azone is one of the most investigated enhancers; it is more effective for hydrophilic drugs than lipophilic drugs. And as a skin permeation enhancer, the efficacy of Azone appears to be dependent on its concentration, and surprisingly, it is more effective at lower concentrations, being employed typically between 0.1 to $5 \%$. However, the lag time of Azone was very high, which is from $2 \mathrm{~h}$ to $10 \mathrm{~h}$, but its effects can last for many days [38].

Azone increases the ability of drugs to permeate the skin by interacting with the lipid domains of the SC, disordering lipid bilayers, disrupting their packing arrangement, and increasing lipid solubilization [38].

\section{The mechanisms of chemical permeation enhancers}

Chemical penetration enhancers can dramatically increase skin permeability by reducing the resistance of the skin to drug diffusion [39-41]. Skin permeation enhancers may exert their effects by one or a combination of the following several possible mechanisms:

1. Interaction with the intercellular lipid matrix (especially cholesterol and ceramides), disrupting the highly ordered lamellar structure.

2. They can interact with intracellular protein (influencing the conformation of keratin in the corneocytes or proteins in desmosomes) to promote permeation of drugs through the corneocyte layer.

3. CPEs can improve the partition of the drugs, increasing the concentration of the drug in the skin.

4. CPEs can dissolve the skin-tissue components [42,43].

\section{Microneedles}

Recently, microneedles (MNs) have gained more and more attention as the excellent candidates for transdermal delivery. MN arrays are composed of multiple micron-sized projections which are typically assembled on one side of a supporting base or patch. These microprojections generally range from lengths as short as $25 \mu \mathrm{m}$ to those as long as $2000 \mu \mathrm{m}$, so that they can create little holes in the stratum corneum without pian and become the effective way to enhance the delivery of therapeutic molecules and macromolecules complexes across biological membranes including mucosal tissue, and skin, sclera [44-46].

Microneedles can be fabricated from different kinds of materials, such as silicon, silicon dioxide, metals such as stainless steel, palladium, nickel and titanium, glass, ceramics and polymers, and they differ in shape and in length. MN have been produced in various geometries. These microstructure geometries can be in the form of needle-like (most common MN geometries which can be sharp-, tapered-, conical- or bevel-tipped), microblades, and blunt-projections or shaped in an arrow-head [47-51].

In addition, MNs as a parenteral route of administration could provide the relatively easy and patient-friendly administration of therapeutics with potential efficacy at low cost. And some studies have shown that MNs can penetrate the skin, cross the stratum corneum into the viable epidermis, and reside primarily in the dermal layer, avoiding contact with blood vessels and nerve fibres. So MNs can provide a pain-free, minimally invasive ways to delivery both small and large molecular weight without bleeding at the application site (Figure 2) [54].

Over the last decade, extensive experiments about MNs has been carried out with the use of a wide range of techniques. It is worth mentioning that the effect of MNs has been demonstrated in in vitro, ex vivo and in vivo experiments. 


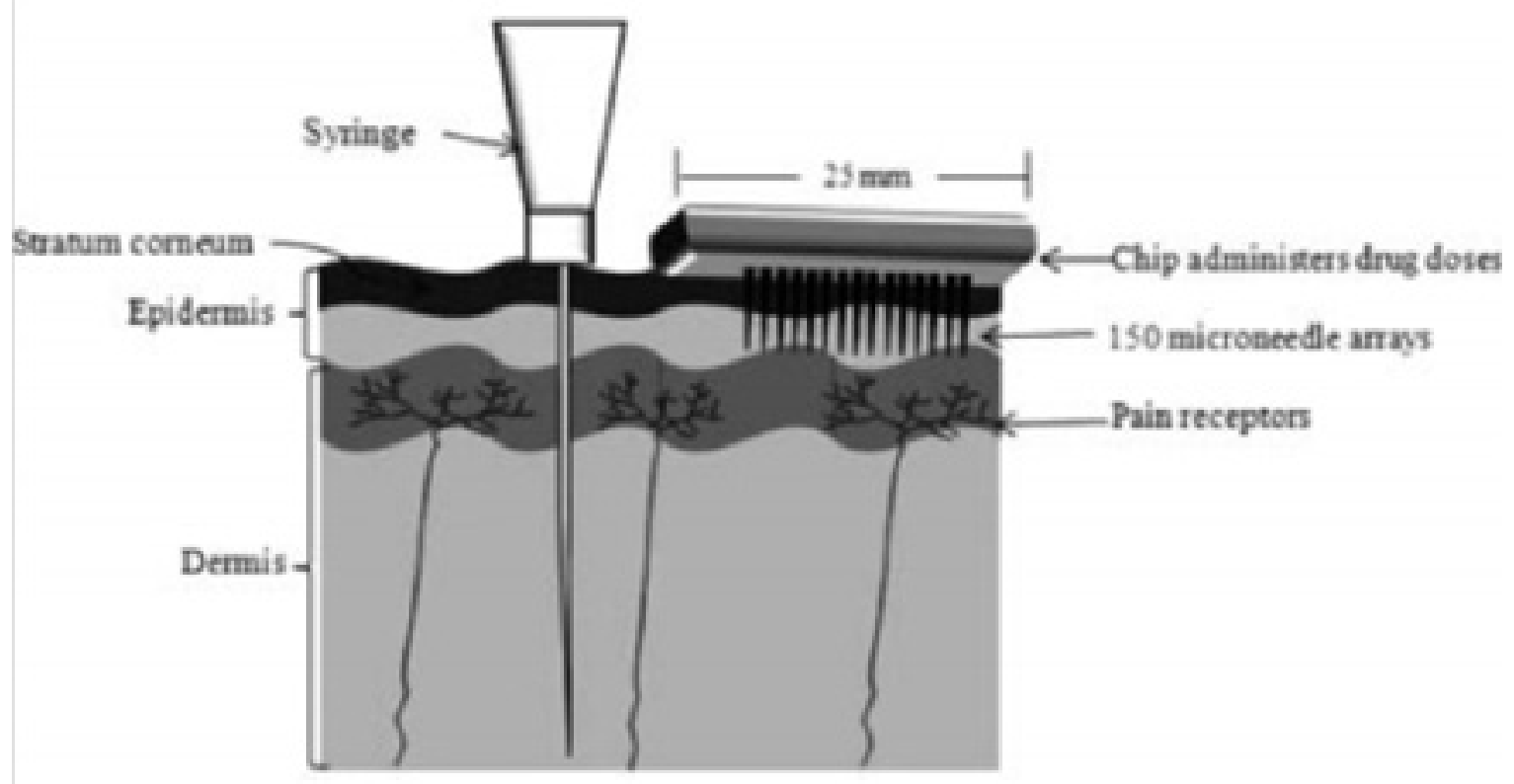

Figure 2: Reference of the comparison of MN arrays and ordinary syringes

\section{The classification of MNs}

$\mathrm{MN}$ arrays can be classified two different types: solid microneedles (Figure 3a,b and c) and hollow microneedles (Figure 3e and f) [51-53], which are commonly described in the literature. As the names imply, the two types of MNs have been used by different approaches for the delivery of therapeutics across the skin.
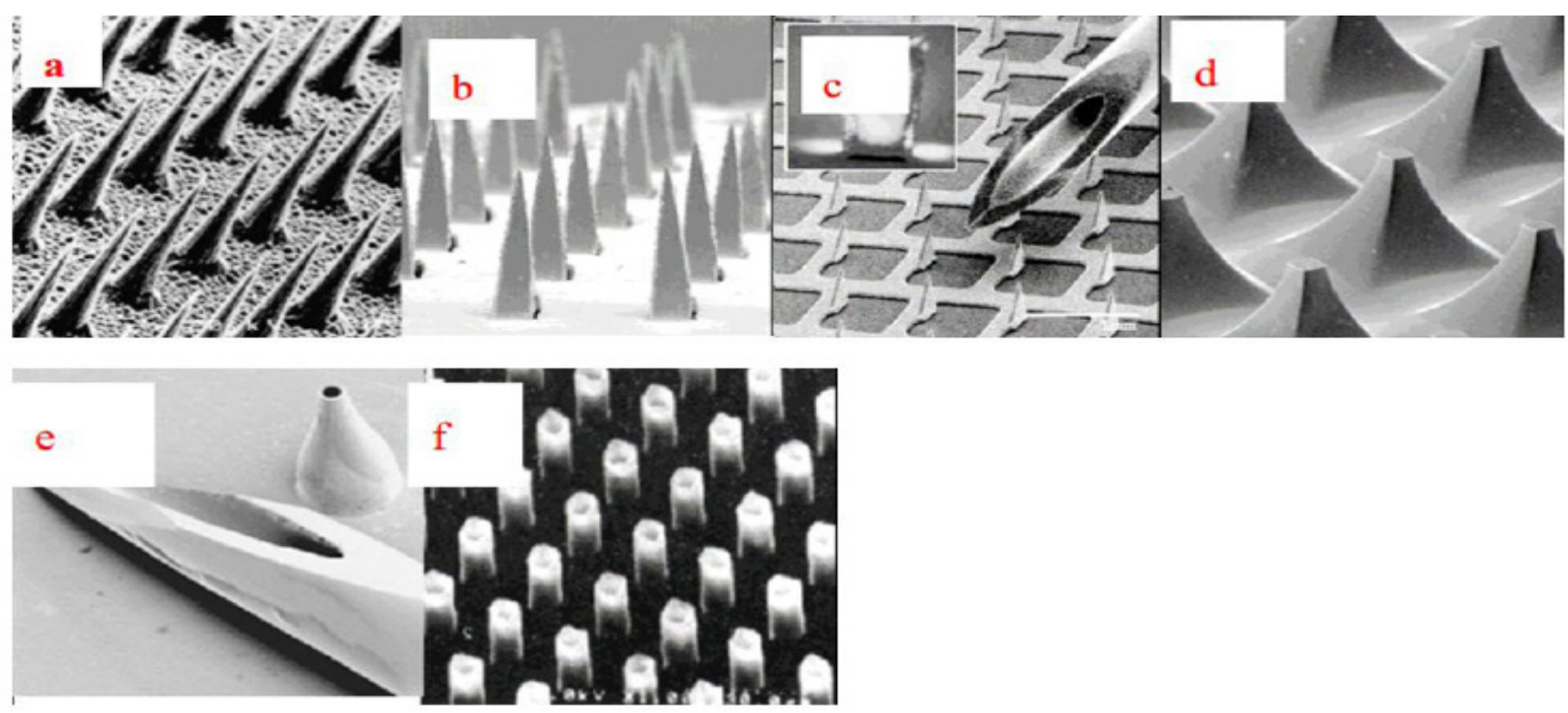

Figure 3: The images of solid microneedles (a,b and c) and hollow microneedles (e and f)

\section{Drug delivery through MNs}

Different types of MNs have different ways of drug delivery. There are four general means to delivery drug by microneedles, as schematically depicted in Figure 4.

Drug delivery by solid microneedles: There are three main approaches which can be used to deliver drugs into the skin by solid microneedles, including the "poke and release" approach, the "poke and patch" approach, and the "coat and poke" approach, as schematically shown in Figure 4a, b and c. 

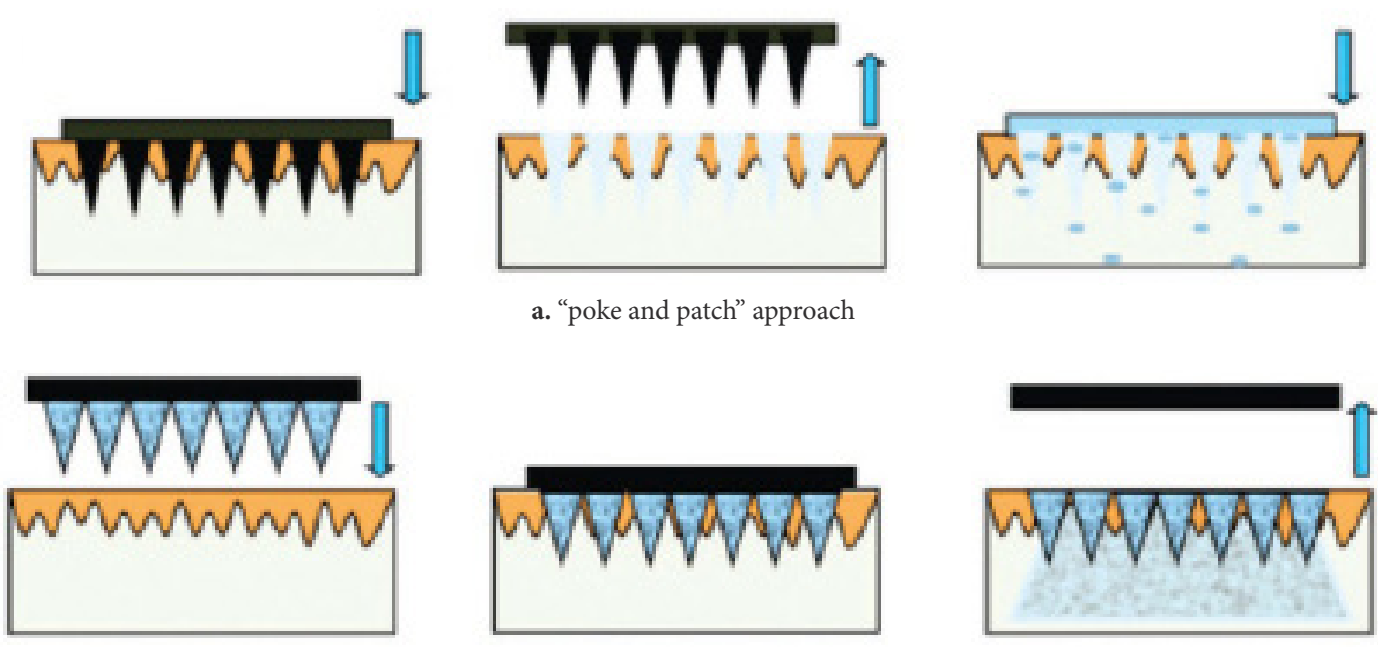

b. "poke and release" approach
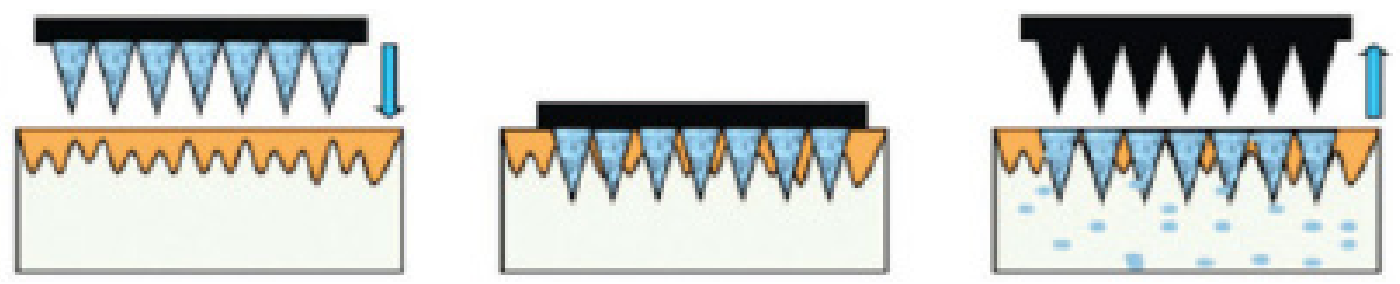

c. the "coat and poke" approach
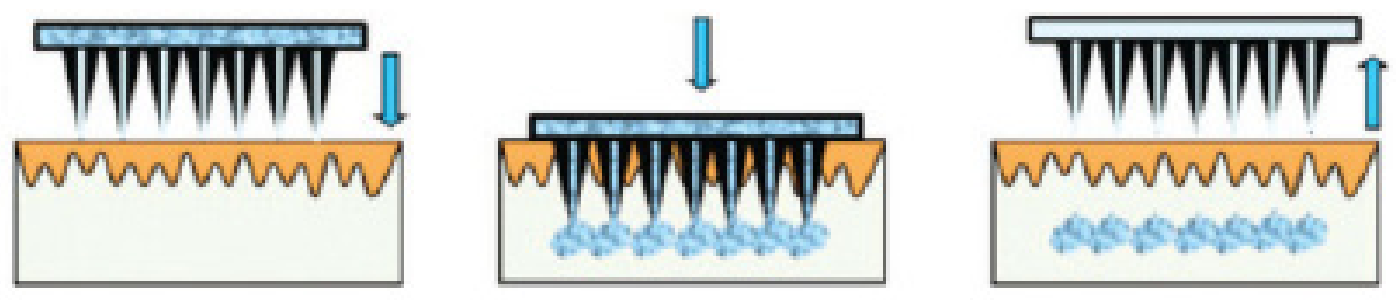

d. "poke and flow"

Figure 4: Reference schematic diagram of the approaches for drug delivery by different designs of microneedles: (a) 'poke and patch' using solid microneedles; (b) 'coat and poke' using coated solid microneedles; (c) 'poke and release' using polymeric microneedles; (d) 'poke and flow' using hollow microneedles

In an early phase of research on microneedles, an array of solid microneedles made up of silicon wafers was used to pierce through skin to overcome the barrier caused by the stratum corneum. Subsequently, the microneedles were removed and transient microchannels were created in the skin upon which a conventional patch containing the drug formulation was applied onto the treated skin surface so that the drug can diffuse to the formed microchannels. This approach is named as "poke and patch", which is the first strategy of MN-mediated transdermal drug delivery [55-57].

Some studies have reported that the permeability for different drugs was increased by "poke and patch" approach up to four orders in magnitude. And for the "poke and patch" approach, the key is that the micropores should stay open conditions during the drug application period. However, it has been demonstrated that the micropores generated by microneedles can keep open conditions until at least $72 \mathrm{~h}$ and this approach may not be applicable, because longer opening times can be along with the risk of infection [58-61].

Subsequent researches in microneedles result in the development of a "poke and release" approach. The second approach for (Trans) dermal drug delivery features a modulated release of the drugs from the microneedles by using a variety of available materials, such as polymers and polysaccharides, which serve as a drug depot and can be either slowly degraded or dissolved after administration. It means that, the microneedles must remain on the application site until the drug is totally released. The administration of a large amount of drug is still not feasible by the "poke and release" approach, which lead to the development of "coat and poke" approach [62]. 
The further current approach for (Trans) dermal drug delivery by solid microneedles is via the "coat and poke" approach. Solid microneedles are coated with drug solution by dip-coating technology, containing DNA, RNA, proteins, hydrophilic and hydrophobic low-molecular-weight drugs, inactivated pathogens, and particles, etc. After the skin was pierced, the drug can be completely released. However, a limited quantity of drugs could be coated over the microneedles (only about $1 \mathrm{mg}$ ) because thick coatings can decrease sharpness of microneedles, resulting in a very low skin delivery efficiency. Besides, uniform coating in the process is the key, which can be achieved by optimizing the experimental conditions. Lastly, during the process of coating, the challenge is the relatively high loss of drug. In order to solve the problem, multiple methods of coating and coating devices have been invented during the past decade [63-65].

Drug delivery by hollow microneedles: Hollow microneedles deliver drugs via a more effective approach (Figure 4d). This approach was known as"poke and flow". After the microneedle system penetrates into the skin, the drug solution was allowed to flow through the pore from the reservoir and release into the skin. The most important advantage of hollow microneedles over the approaches above-mentioned is the possibility to facilitate fluid flow by the driving forces, such as pressure, thus a large amount of drug can be administered by hollow microneedles, thereby allowing higher rates of drug delivery [66,67]. In general, to prevent blockage of channel and insert the microneedle easily, the pore is kept alongside walls instead of at the centre.

In order to improve the permeability of insulin unilamellar, Chen et al. researched the delivery efficiency of the drug through hollow microneedles combined with iontophoresis so the delivery of drug can be in a controlled way by regulating the current. The current may be turned on and off by the patient based on their own needs and desires. Even though the combination of hollow microneedles and iontophoresis can increase the delivery efficiency, this leads to skin irritation when a constant current is used [68].

Although the design of the microneedle is more effective to achieve drug delivery compared with other approaches, and the microneedle can be used to deliver drugs either at a local site or systemically and do not cause any pain or irritation, the disadvantages of microneedle system are that: (1) a small amount of drug can be given (less than 1mg); (2) the accuracy of dosage may be less than hypodermic needles; (3) the hollow microneedles must be not blocked and broken off; (4) repetitive injection may destroy the veins; (5) the external environment, such as hydration of the skin, could affect delivery.

\section{Iontophoresis}

Iontophoresis, which is defined as "the application of a small electric current $\left(0.5 \mathrm{~mA} / \mathrm{cm}^{2}\right.$ or less $)$ with a low voltage, to drive ionic and polar molecules across the skin and into the tissues", has been used to improve the transdermal delivery of drugs, such as neutral and charged molecules, low and high molecular weight drugs namely phenobarbital, ranitidine and zidovudine. Basically, Iontophoresis consists of two electrodes: a cathode and an anode. Commonly, the small electric current is driven from an external supply into the skin at the anode, and back out at the cathode (Figure 5). Direct current iontophoresis with a constant current approach is the most common form of iontophoretic transport. Alternating current has also been employed in transdermal iontophoretic drug delivery. Some reports has suggested that alternating current compared to direct current can reduce skin irritation, eliminate potential electrochemical burns during long iontophoresis application. Under the circumstances when iontophoresis is used, the direct current or alternating current electric field reduces skin electrical resistance by the mechanism of electroporation. This electroporation effect has been found to dramatically increase as the voltage is increased. Because iontophoresis has many advantages, such as ease of application, high efficiency, safety and miniaturized instrument, it is known as a promising approach to improve transdermal drug delivery $[68,69]$.

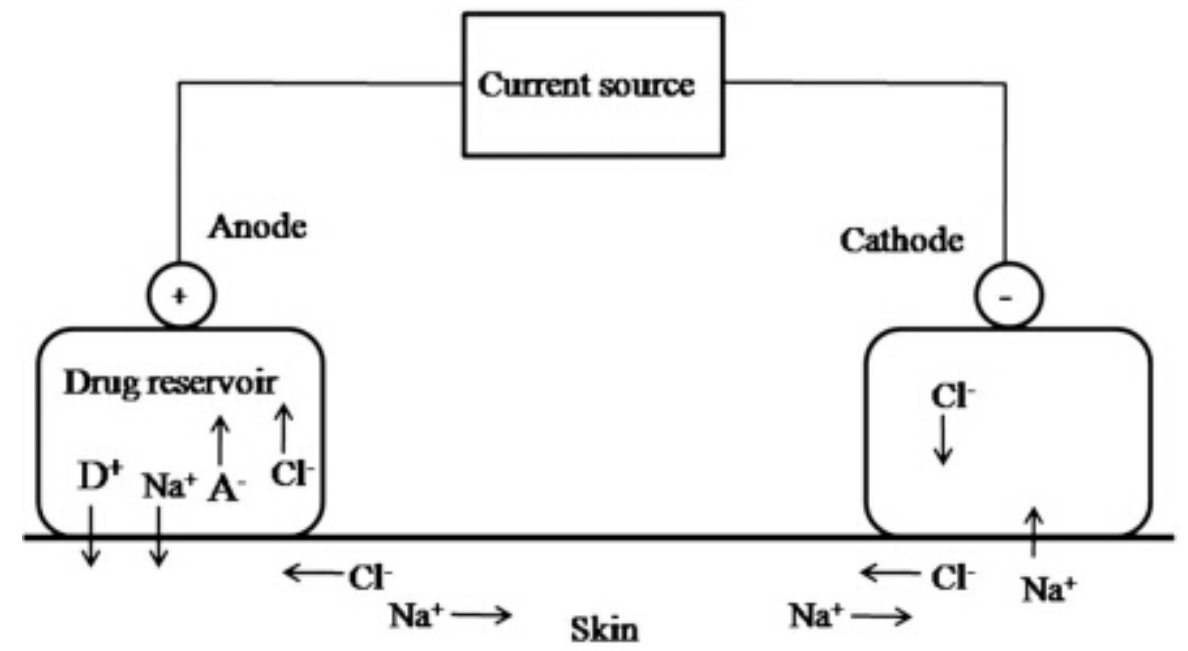

Figure 5: Reference schematic diagram of the sketch of iontophresis using an $\mathrm{Ag} / \mathrm{AgCl}$ electrode system: $\mathrm{Na}^{+}=$sodium ion in drug reservoir, $\mathrm{Cl}^{-}=$chloride ions in drug reservoir or from $\mathrm{AgCl}$ electrode, $\mathrm{A}^{-}=$anionic species of drug, $\mathrm{D}^{+}=$cationic species of drug. 
However, several limitations and disadvantages have been associated with the use of such approach, including stability, loadability, handleability. Besides, chemical and thermal stability of the reservoir and the electrode is one of the considering problems.

\section{The mechanisms of iontophoresis}

The mechanisms of transdermal iontophoresis include electroosmosis (electric field induced solvent flow), electromigration (electric field charge interaction) and electroporation (increasing the porosity of skin due to electric field).

Electroosmosis: Transdermal iontophoresis can enhance the transport of ionic drugs by the imposed electric field or voltage. The electric field imposes an additional force, and then drives the ion through the skin more efficiently than the pure diffusion or "passive" transdermal drug delivery. In general, if the drug is not ionogenic, there is no direct interaction between the drug and the electric field, so drug delivery should not be improved. However, some studies have demonstrated that electroosmosis can cause bulk solvent flow, which is suggested as the possible mechanism for the promoted permeability of neutral species.

Electroosmosis refers to the relative movement between liquid (usually water) and the fixed solid phase in the electric field. Electroosmotic flow is referred to bulk flow of the solution or volume flow, which occurs in a wide variety of membrane systems containing fixed charges. This flow is not diffusion, which has nothing to do with concentration gradients. The direction of flow is always in the direction of counterion flow, which may be against or with the current flow, depending on the nature of the membrane systems [70-72].

Electroporation: Electroporation utilizes short, high-voltage electrical pulses (50 to 2000V) to create temporary pores or aqueous pathways within the SC and promote transdermal drug delivery, which are efficient for DNA or large hydrophilic drug molecules delivery. The reason is that electroporation results in fluidization of lipids and phase transition because of joule heating and higher localized temperature (over $60^{\circ} \mathrm{C}$ ). The main modes of electroporation are diffusion and electrophoresis. Under the action of highvoltage electrical pulses, the transdermal transport of drugs occurs via localized regions of the stratum corneum. In these regions, the small molecule drugs can be transported via the transcellular route in the condition of short high-voltage electrical pulses, but the intercellular route possibly with appendageal pathway is adopted when lower voltage and prolonged pulse duration are used.

Electroporation can be used together with chemical enhancers such as magnesium chloride, calcium chloride, calcium bromide, heparin, urea, anionic phospholipids and sodium thiosulfate. These chemical enhancers such as magnesium chloride can interact with the lipid bilayers of SC and overcome the skin barrier with slow recovery under the influence of electroporation. However not all the chemical enhancers can disrupt the lipid lamellae. Instead, they insert themselves into the aqueous pathways or pores, and then stabilize the transient disruption created by electroporation. The combination of chemical enhancer and electroporation will enlarge the aqueous pores and prolong the lifespan of them [73-76].

Electromigration: The electroosmosis can be useful for both charged and neutral molecules whereas electromigration is primarily used to drive the charged molecules across the skin. Under the influence of electric current, the appendageal pathway predominates when the ions tend to travel the path which offers the least resistance. On the other hand, the non-appendageal pathway has also been identified as an important player in iontophoretic delivery when the intercellular route is concerned. The intercellular route consists of polar regimes in lipid lamellae. When electrical current is imposed, these polar aqueous pores are formed as a result of flip-flop movement of polypeptide helices in the stratum corneum. They confer an alternative pathway for drug transport [77-79].

\section{Iontophoresis - Microneedle Combinations}

Nowdays, it has been confirmed that MN will result in creating of aqueous pathways of low electrical resistance. The synergistic effect of iontophoresis and $\mathrm{MN}$ technologies can lead to an increased rate of transdermal delivery for a range of drug molecules with the benefit of precise electronic control. In order to date the combination of iontophoresis and MNs, some researchers have focused on either the use of an electric stimulus to promote drugs to move through the central bore of hollow MNs or the use of non drugloaded solid MNs (used to merely puncture the skin) before the application of an electrically conducting drug formulation. In these cases, the central bore of the hollow MNs may become blocked and the pores created by the solid MNs could be likely to close, and the use of iontophoresis may stimulate drugs to move. The use of hydrogel- forming MN or drug-loaded dissolving polymer MN systems may overcome some of the above mentioned problems and thus allow the combination of MN and iontophoresis to enhance transdermal delivery of a wide range of drug molecules [80-84].

\section{Conclusion}

Transdermal drug delivery is an alternative and promising way to systematic administration of drugs on account of the validated therapeutic effect, safety, low cost and convenient use. To over the barrier and improve transdermal efficiency, these methods mentioned above have been deeply investigated and tested in a number of humans clinical trials and they enable patient-controlled dosing and more complex delivery profiles than passive transdermal drug delivery systems. 


\section{Acknowledgement}

This work was supported by the Foundation of Jinan Science and Technology Development Program (201303055). This project was supported by Project of Shandong Province Higher Educational Science and Technology Program (J14LK61).

\section{References}

1. Patil UK, Saraogi R (2014) Natural products as potential drug permeation enhancer in transdermal drug delivery system. Arch Dermatol Res 306: 419-26. DOI $10.1007 / \mathrm{s} 00403-014-1445-\mathrm{y}$.

2. Lam PL, Gambari R (2014) Advanced progress of microencapsulation technologies: In vivo and in vitro models for studying oral and transdermal drug deliveries. J Cont Rel 178: 25-45.

3. Prausnitz MR, Mitragotri S, Langer R (2004) Current status and future potential of transdermal drug delivery. Nat Rev Drug 3: 115-24.

4. Baviskar DT, Parik VB, Gupta HN, Maniyar AH, Jain DK (2012) Design and Evaluation of Patches for Transdermal Delivery of Losartan Potassium. PDA J Pharm Sci Technol 66: 126-35.

5. Azeem A, Khan ZI, Aqil M, Ahmad FJ, Khar RK, et al. (2009) Microemulsions as a surrogate carrier for dermal drug delivery. Drug Dev Ind Pharm 35: 525-47.

6. Khafagy el-S, Morishita M, Onuki Y, Takayama K (2007) Current challenges in non-invasive insulin delivery systems:A comparative review. Adv Drug Deliv Rev 59: $1521-46$.

7. Prow TW, Grice JE, Lin LL, Faye R, Butler M, et al. (2011) Nanoparticles and microparticles for skin drug delivery. Adv Drug Deliv Rev 63: 470-91.

8. Prausnitz MR (2001) Overcoming skin's barrier: the search for effective and user-friendly drug delivery. Diabetes Technol Ther 3: 233-6.

9. Roberts MS, Cross SE, Pellett MA (2002) Skin transport Dermatological and Transdermal Formulations. Marcel Dekker Inc 14: 89-195.

10. Barry BW (1991) Modern methods of promoting drug absorption through the skin. Mol Aspects Med 12: 195-241.

11. Barry BW (1983) Dermatological formulation: Percutaneous absorption. Mercel Dekker 18: 25-238.

12. Purdon CH, Azzi CG, Zhang J, Smith EW, Maibach HI (2004) Penetration enhancement of transdermal delivery-current permutations and limitations. Crit Rev Ther Drug Carr Syst 21: 97-132.

13. Benson HA (2005) Transdermal drug delivery: penetration enhancement techniques. Curr Drug Deliv 2: 23-33.

14. Kanikkannan N, Babu RJ, Singh M (2006) Structure-activity relationship of chemical penetration enhancers. Adv Drug Deliv Rev 56: 603-18.

15. William AC, Barry BW (2004) Penetration enhancers. Adv Drug Deliv Rev 56: 603-18.

16. Ogiso T, Shintani M (1990) Mechanism for the enhancement effect of fatty acids on the percutaneous absorption of propranolol. J Pharm Sci 79: 1065-71.

17. Kandimalla K, Kanikkannan N, Andega S, Singh M (1999) Effect of fatty acids on the permeation of melatonin across rat and pig skin in-vitro and on the transepidermal water loss in rats in-vivo. J Pharm Pharmacol 51: 783-90.

18. Florence AT, Tucker IG, Walters KA (1984) In Structure Performance Relationships in Surfactants. American Chemical Societv 65: 189-207.

19. Guy RH, Hadgraftj J, Maibach HJ (1985) Mechanism for the enhancement effect of fatty acids on the percutaneous absorption of propranolol. Toxicol Appl Pharmacol 78: 123-9.

20. Aungst BJ, Rogers NJ, Shefter E (1986) Enhancement of naloxone penetration through human skin in vitro using fatty acids, fatty alcohols, surfactants, sulfoxides and amines. Int J Pharm 33: 225-34.

21. Komata Y, Inaoka M, Kaneko A, Fujie T (1992) In vitro percutaneous absorption of thiamine disulfide from a mixture of propylene glycol and fatty acid. J Pharm Sci 81: 744-6.

22. Aungst BJ (1989) Structure/effect studies of fatty acid isomers as skin penetration enhancers and skin irritants. Pharm Res 6: 244-7.

23. Ongpipattanakul B, Burnette RR, Potts RO, Francoeur ML (1991) Evidence that oleic acid exists in a separate phase within stratum corneum lipids. Pharm Res 8: $350-4$.

24. Sinha VR, Kaur PM (2000) Permeation enhancers for transdermal drug delivery. Drug Dev Ind Pharm 26: 1131-40.

25. Barry BW, Williams AC (1993) Terpenes as skin penetration enhancers. Marcel Dekker 9: 95-111.

26. Williams AC, Barry BW (1991) Terpenes and the lipid-protein-partitioning theory of skin penetration enhancement. Pharm Res 8: 17-24.

27. Vaddi HK, Ho PC, Chan SY (2002) Terpenes in propylene glycol as skin-penetration enhancers: permeation and partition of haloperidol, Fourier transform infrared spectroscopy and differential scanning calorimetry. J Pharm Sci 91: 1639-51.

28. Asbill CS, El-Kattan AF, Michniak B (2000) Enhancement of transdermal drug delivery: chemical and physical approaches. Crit Rev Ther Drug Carrier Syst 17: $621-58$.

29. Krishnaiah YS, Satyanarayana V, Bhaskar P (2003) Influence of menthol and pressure-sensitive adhesives on the in vivo performance of membrane-moderated transdermal therapeutic system of nicardipine hydrochloride in human volunteers. Eur J Pharm Biopharm 55: 329-37.

30. Okabe H, Obata Y, Takayama K, Nagai T (1990) Percutaneous absorption enhancing effect and skin irritation of monocyclic monoterpenes. Drug Des Deliv 6: $229-38$.

31. Barry BW (1998) The LPP theory of skin penetration enhancement. Maturitas 29: 165-85.

32. Ghafourian T, Zandasrar P, Hamishekar H, Nokhodchi A (2004) The effect of penetration enhancers on drug delivery through skin: a QSAR study. J Control Release 99: 113-25.

33. Montia D, Saettone MF, Giannaccini B, Angeli DG (1995) Enhancement of transdermal penetration of dapiprazole through hairless mouse skin. J Control Release 33: 71-7. 
34. Okabe H, Takayama K, Ogura A, Nagai T (1989) Effect of limonene and related compounds on the percutaneous absorption of indomethacin. Drug Des Deliv 4: 313-21.

35. Narishetty ST, Panchagnula R (2004) Transdermal delivery of zidovudine: effect of terpenes and their mechanism of action. J Control Release 95: 367-79.

36. Kang L, Yap CW, Lim PF, Chen YZ, Ho PC, et al. (2007) Formulation development of transdermal dosage forms: quantitative structure activity relationship model for predicting activity of terpenes that enhance drug penetration through human skin. J Control Release 120: 211-9.

37. Williams AC, Barry BW (2004) Penetration enhancers. Adv Drug Deliv Rev 56: 603-18.

38. Gallarate M, Gasco MR, Trotta M, Chetoni P, Saettone MF (1993) Preparation and evaluation in-vitro of solutions and o/w microemulsions containing levobunolol as ion-pair. Int J Pharm 100: 219-25.

39. Barry BW (1987) Mode of action of penetration enhancers in human skin. J Control Release 6: 85-97.

40. Walters KA, Brain KR, Green DM, James VJ, Watkinson AC, et al. (1998) Comparison of the transdermal delivery of estradiol from two gel formulations. Maturitas 29: 189-95.

41. Barry BW (1991) Lipid-protein-partitioning theory of skin penetration enhancement. J Control Release 15: 237-48.

42. Menon GK, Lee SH, Roberts MS (1998) Ultrastructural effects of some solvents and vehicles on the stratum corneum and other skin components: Evidence for an "extended mosaic-partitioning model of the skin barrier". J Control Release 8: 727-51.

43. Guyn RH, Hadgraft J (1987) The effect of penetration enhancers on the kinetics of percutaneous absorption. J Control Release 5: 43-51.

44. Gill HS, Prausnitz MR (2007) Coated microneedles for transdermal delivery. J Control Release 117: 227-37.

45. Teo MA, Shearwood C, Ng KC, Lu J, Moochhala S (2005) In vitro and in vivo characterization of MEMS microneedles. Biomed Microdevices 7: 47-52.

46. Wermeling DP, Banks SL, Huclson DA, Gill HS, Gupta J, et al. (2008) Microneedles permit transdermal delivery of a skin-impermeant medication to humans. Proc Natl Acad Sci 105: 2058-63.

47. Li WZ, Huo MR, Zhou JP, Zhou YQ, Hao BH, et al. (2010) Super-short solid silicon microneedles for transdermal drug delivery applications. Int J Pharm 389: $122-9$.

48. Parker ER, Rao MP, Turner KL, Meinhart CD, MacDonald NC (2007) Bulk micromachined titanium microneedles. J Microelectromech Syst 16: $289-95$.

49. Noh YW, Kim TH, Baek JS, Park HH, Lee SS, et al. (2010) In vitro characterization of the invasiveness of polymer microneedle against skin. Int J Pharm 397: 201-5.

50. Bystrova S, Luttge R (2011) Micromolding for ceramic microneedle arrays. Microelectron Eng 88: 1681-4.

51. Prausnitz MR (2004) Microneedles for transdermal drug delivery. Adv Drug Del Rev 56: 581-7.

52. Mukerjee EV, Collins SD, Isseroff RR, Smith RL (2004) Microneedle array for transdermal biological fluid extraction and in situ analysis. Sens Actuators A 114: 267-75.

53. Barry BW (2001) Novel mechanisms and devices to enable successful transdermal drug delivery. Eur J Pharm Sci 14: 101-14.

54. Bal SM, Caussin J, Pavel S, Bouwstra JA (2008) In vivo assessment of safety of microneedle arrays in human skin. Eur J Pharm Sci 35: 193-202.

55. Prausnitz MR, Gill HS, Park JH (2008) Modified Release Drug Delivery (2 ${ }^{\text {nd }}$ edn) Healthcare, New York, USA 295-309.

56. Banga AK (2009) Microporation applications for enhancing drug delivery. Expert Opin Drug Deliv 6: 343-54.

57. Sivamani RK, Liepmann D, Maibach HI (2007) Microneedles and transdermal applications. Expert Opin Drug Deliv 4: 19-25.

58. Henry S, McAllister DV, Allen MG, Prausnitz MR (1998) Microfabricated microneedles: a novel approach to transdermal drug delivery. J Pharm Sci 87: 922-5. 59. Kalluri H, Kolli CS, Banga AK (2011) Characterization of microchannels created by metal microneedles: formation and closure. AAPS J 13: 473-81.

60. Kalluri H, Banga AK (2011) Formation and closure of microchannels in skin following microporation. Pharm Res 28: 82-94.

61. Chen H, Zhu H, Zheng J, Mou D, Wan J, et al. (2009) Iontophoresis-driven penetration of nanovesicles through microneedle-induced skin microchannels for enhancing transdermal delivery of insulin. J Control Release 139: 63-72.

62. Chen B, Wei J, Tay FEH, Wong YT, Iliescu C (2008) Silicon microneedle array with biodegradable tips for transdermal drug delivery. MicrosystTechnol 14: $1015-9$.

63. McGrath MG, Vrdoljak A, O'Mahony C, Oliveira JC, Moore AC, et al. (2011) Determination of parameters for successful spray coating of silicon microneedle arrays. Int J Pharm 415: 140-9.

64. Chen X, Prow TW, Crichton ML, Jenkins DW, Roberts MS, et al. (2009) Dry-coated microprojection array patches for targeted delivery of immunotherapeutics to the skin. J Control Release 139: 212-20.

65. Xie Y, Xu B, Gao Y (2005) Controlled transdermal delivery of model drug compounds by MEMS microneedle array. Nanomedicine 1: 184-90.

66. Milewski M, Brogden NK, Stinchcomb AL (2010) Current aspects of formulation efforts and pore lifetime related to microneedle treatment of skin. Expert Opin Drug Deliv 7: 617-29.

67. Bal SM, Ding Z, van Riet E, Jiskoot W, Bouwstra JA (2010) Advances in transcutaneous vaccine delivery: do all ways lead to Rome? J Control Release 148: $266-82$.

68. Djabri A, Guy RH, Delgado-Charro MB (2012) Passive and iontophoretic transdermal delivery of phenobarbital: Implications in paediatric therapy. Int J Pharm 435: 76-82.

69. Oh SY, Jeong SY, Park TG, Lee JH (1998) Enhanced transdermal delivery of AZT (Zidovudine) using iontophoresis and penetration enhancer. J Control Release 51:161-8.

70. Langer R (2004) Transdermal drug delivery: past progress, current status, and future prospects. Adv Drug Deliv Rev 56: 557-8. 
71. Gangarosa LP, Park NH, Wiggins CA, Hill JM (1980) Increased penetration of non-electrolytes into mouse skin during iontophoretic water transport (iontohydrokinesis). J Pharmacol Exp Ther 212: 377-81.

72. Burnette RR, Marrero D (1986) Comparison between the iontophoretic and passive transport of thyrotropin releasing hormone across excised nude mouse skin. J Pharm Sci 75: 738-43.

73. Burnette RR, Ongpipattanakul B (1987) Characterization of the permselective properties of excised human skin during iontophoresis. J PharmSci 76: 765-73.

74. Denet AR, Vanbever R, Préat V (2004) Skin electroporation for transdermal and topical delivery. Adv Drug Deliv Rev 56: 659-74.

75. Sen A, Daly ME, Hui SW (2002) Transdermal insulin delivery using lipid enhanced electroporation. Biochim Biophys Acta 1564: 5-8.

76. Tokudome Y, Sugibayashi K (2003) The synergic effects of various electrolytes and electroporation on thein vitro skin permeation of calcein. J Control Release 92: 93-101.

77. Tokudome Y, Sugibayashi K (2004) Mechanism of the synergic effects of calcium chloride and electroporation on the in vitro enhanced skin permeation of drugs. J Control Release 95: 267-74.

78. Mitragotri S (2013) Devices for overcoming biological barriers: the use of physical forces to disrupt the barriers. Adv Drug Deliv Rev 65: 100-3.

79. Calatayud-Pascual MA, Balaguer-Fernández C, Serna-Jiménez CE, Del Rio-Sancho S, Femenía-Font A, et al. (2011) Effect of iontophoresis on in vitro transdermal absorption of almotriptan. Int J Pharm 416: 189-94.

80. Panchagnula R, Pillai O, Nair VB, Ramarao P (2000) Transdermal iontophoresis revisited. Curr Opin Chem Biol 4: 468-73.

81. Lanke SS, Kolli CS, Strom JG, Banga AK (2009) Enhanced transdermal delivery of low molecular weight heparin by barrier perturbation. Int J Pharm 365: 26-33.

82. Wang Y, Thakur R, Fan Q, Michniak B (2005) Transdermal iontophoresis: combination strategies to improve transdermal iontophoretic drug delivery. Eur J Pharm Biopharm 60: 179-91.

83. Katikaneni S, Badkar A, Nema S, Banga AK (2009) Molecular charge mediated transport of a 13 kD protein across microporated skin. Int J Pharm 378: 93-100.

84. Wu XM, Todo H, Sugibayashi K (2007) Enhancement of skin permeation of high molecular compounds by a combination of microneedle pretreatment and iontophoresis. J Control Release 118: 189-95.

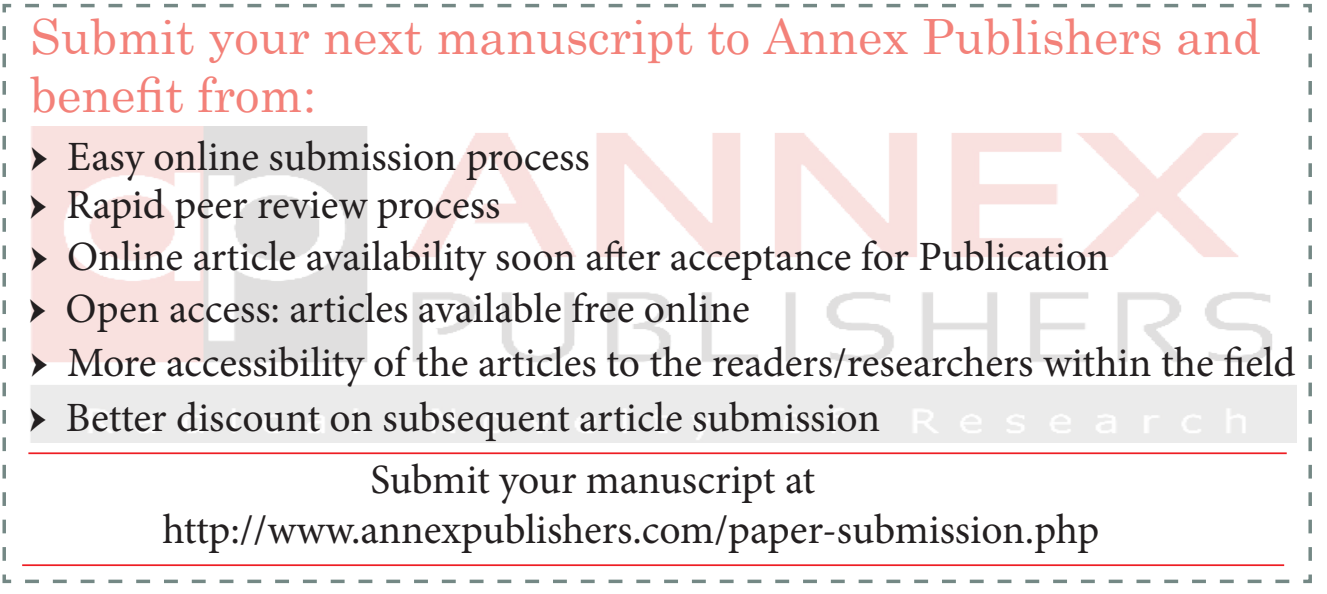

